Pacific Journal of Mathematic 


\title{
SETS GENERATED BY RECTANGLES
}

\author{
R. H. Bing, W. W. Bledsoe, \\ AND R. D. MAULDIN
}

For any family $F$ of sets, let $\mathscr{B}(F)$ denote the smallest $\sigma$-algebra containing $F$. Throughout this paper $X$ denotes a set and $\mathscr{R}$ the family of sets of the form $A \times B$, for $A \subseteq X$ and $B \subseteq X$. It is of interest to find conditions under which the following holds:

(1) Each subset of $X \times X$ is a member of $\mathscr{B}(\mathscr{P})$

The interesting case is when

$$
\omega_{1}<\operatorname{Card} X \leqq c,
$$

since results for other cases are known.

It is shown in Theorem 9 that (1) is equivalent to

There is a countable ordinal $\alpha$ such that each subset of $X \times X$ can be generated from $\mathscr{R}$ is $\alpha$ Baire process steps .

It is also shown that the two-dimensional statements (1) and (2) are equivalent to the one-dimensional statement

There is a countable ordinal $\alpha$ such that for each family $H$ of subsets of $X$ with

(3) $\quad$ Card $H=$ Card $X$, there is a countable family $G$ such that each member of $H$ can be generated from $G$ in $\alpha$ steps .

It is shown in Theorem 5 that the continuum hypothesis $(\mathrm{CH})$ is equivalent to certain statements about rectangles of the form (1) and (2) with $\alpha=2$.

Rao [7, 8] and Kunen [2] have shown that

THEOREM 1. If Card $X \leqq \omega_{1}$ (the first uncountable cardinal) then (1) is true and if Card $X>c$ then (1) is false.

The question of whether (1) is true (without the requirement Card $X \leqq \omega_{1}$ ) was raised by Johnson [1] and earlier by Erdös, Ulam, and others (see [8], p. 197). The arguments in Kunen's thesis actually showed that if Card $X \leqq \omega_{1}$ then

Each subset of $X \times X$ can be generated from $\mathscr{R}$ in 2 steps (i.e., each subset is a member of $\mathscr{R}_{\sigma o}$. See definitions in $\S 2$.).

In Theorem 5 we generalize Theorem 1 and Kunen's result (4), 
and give a new characterization of $\mathrm{CH}$ by showing it to be equivalent to certain statements about rectangles of the form (1) and (4).

If $\mathrm{CH}$ is assumed the $\alpha$ appearing in statements (2) and (3) above is 2 (see Theorem 10). This raises the intriguing (but unanswered) question of whether $\alpha$ must always be 2 if (1) holds and $\mathrm{CH}$ is false.

It would also be interesting to know whether statements (1), (2), and (3) are equivalent to statement (5) below. Clearly (3) imples (5).

\section{If $H$ is a family of subsets of $X$ with}

Card $H=\operatorname{Card} X$, then there is a countable family $G$ for which $H \subseteq \mathscr{B}(G)$.

The equivalence of (1) and (2) means for example, (assuming $\mathrm{CH}$ ), that there is a countable family $G$ from which all real Borel sets (or analytic sets, or projective sets) can be generated in two steps (i.e., Borel sets $\left.\leqq G_{\sigma \delta}\right)$. This is remarkable in view of the well known result $[4,8]$ that if $G$ is a countable basis for the real topology, then the Borel sets cannot be generated from $G$ in less than $\omega_{1}$ steps.

As a generalization of this well known result we show in Theorem 12 that any countable family $G$ which is closed to complementation and which generates the Borel sets (i.e., Borel sets $\subseteq \mathscr{B}(G)$ ) must have order $\omega_{1}$. That is

$$
\mathscr{B}(G) \nsubseteq G_{\alpha}
$$

for any countable ordinal $\alpha$. Thus, even though $G$ might generate the Borel sets in $\alpha$ steps (or 2 steps if $\mathrm{CH}$ is assumed), the process, nevertheless, continues to produce new members of $\mathscr{S}(G)$ until we reach $G_{\omega_{1}}$.

We would like to point out in conjunction with our characterization of $\mathrm{CH}$ that Kunen [2] has proved that if Martin's Axiom A holds (see [6]) and Card $X \leqq c$ then (4) holds. He also proved that if $\omega_{1}<$ Card $X \leqq c$ then (1) is independent of ZFC (Zermo-Frankel Axioms + the Axiom of Choice) together with the negation of $\mathrm{CH}$.

2. Notation and definitions. If $G$ is any family of sets, let $G_{0}$ be the family $G$, and for each ordinal $\alpha, \alpha>0$, let $G_{\alpha}$ be the family of all countable unions (intersections) of sets in $\bigcup_{i<\alpha} G_{r}$, if $\alpha$ is odd (even). Limit ordinals will be considered even. (Compare Kuratowski [3].) Thus we have

$$
G_{0}=G, G_{1}=G_{o}, G_{2}=G_{\sigma o}, G_{3}=G_{\sigma \dot{\delta} \sigma}, \cdots, G_{\alpha}, \cdots .
$$

Also $G_{\alpha} \subseteq G_{\alpha+1}$ for each ordinal $\alpha$ and $G_{\omega_{1}}=G_{\omega_{1}+1}$, where $\omega_{1}$ is the first uncountable ordinal. If $\alpha>0$, then the family $G_{\alpha}$ is closed under countable unions (intersections) if $\alpha$ is odd (even). 
We define the order of $G$ to be the first ordinal $\alpha, \alpha>0$, such that $G_{\alpha+1}=G_{\alpha}$.

For each $A \subseteq X$ (or $A \subseteq X \times X$ ), let $A^{\prime}$ be the complement of $A$ with respect to $X$ (or $X \times X$ ), and for each family $G$ of subsets of $X$ (or $X \times X$ ) let $\mathscr{C}(G)$ be the family of complements of $G$. Note that if $\mathscr{C}(G) \subseteq G$, or even if $\mathscr{C}(G) \subseteq G_{\omega_{1}}$, then the family $G_{\omega_{1}}$ is the family $\mathscr{\not}(G)$, the $\sigma$-algebra generated by $G$. Thus, since

$$
(A \times B)^{\prime}=A \times B^{\prime} \cup A^{\prime} \times X \in \mathscr{R}_{1},
$$

it follows that $\mathscr{R}_{\omega_{1}}=\mathscr{B}(\mathscr{R})$.

If $G$ is a family of subsets of $X$, let $V G=\{A \times B: A \subseteq X, B \in G\}$, and let $H G=\{A \times B: A \in G, B \subseteq X\}$.

If $Z \subseteq X \times X$ and $x \in X$, let $Z_{x}$ denote the vertical section of $Z$ at $x, Z_{x}=\{y:(x, y) \in Z\}$.

3. Results. The following lemma is easily proved by transfinite induction.

Lemma 2. If $1 \leqq \alpha<\omega_{1}$ and $A \in G_{\alpha}$, then there is a set $B$ in $G_{1}$ such that $A \subseteq B$.

THEOREM 3. If $G$ is a countable family of subsets of $X, Z \subseteq X \times X$, and $0<\alpha<\omega_{1}$, then $Z \in(V G)_{\alpha}$ if and only if $Z_{x} \in G_{\alpha}$ for each $x \in$ domain $Z$.

Proof. By considering the natural projections of the sets involved on the second coordinate axis, it is easily seen that

$$
\text { if } Z \in(V G)_{\alpha} \text {, then } Z_{x} \in G_{\alpha} \text { for each } x \in \text { domain } Z \text {. }
$$

Now suppose that $Z_{x} \in G_{\alpha}$, for each $x \in \operatorname{domain} Z$, and let $G=$ $\left\{\theta_{1}, \theta_{2}, \theta_{3}, \cdots\right\}$. We complete the proof by transfinite induction on $\alpha$.

Case 1. $\alpha=1$.

For each $n$, let $A_{n}=\left\{x \in \operatorname{domain} Z: \theta_{n} \subseteq Z_{x}\right\}$, and let $Z_{n}=A_{n} \times \theta_{n}$. Then $Z_{n} \in V G$, for each $n$, and

$$
Z=\bigcup_{n=1}^{\infty} Z_{n} \in(V G)_{1} .
$$

Now suppose $1<\alpha<\omega_{1}$, and that the theorem holds for every $\gamma, 0<\gamma<\alpha$.

Case 2. $\alpha$ is even. 
Let $\left\{\gamma_{n}\right\}_{n=1}^{\infty}$ be a sequence of odd ordinals less than $\alpha$ such that each odd ordinal less than $\alpha$ appears infinitely often in $\left\{\gamma_{2}\right\}_{n=1}^{\infty}$. For each $x \in$ domain $Z$, let

$$
D_{1}(x), D_{2}(x), D_{3}(x), \cdots
$$

be a sequence such that $D(x) \in G_{r_{i}}$ for each $i$, and

$$
Z_{x}=\bigcap_{i=1}^{\infty} D_{i}(x) \text {. }
$$

This can be done in view of Lemma 2. For each $i$, let

$$
Z^{i}=\bigcup_{x \in \operatorname{lioma\operatorname {ln}} Z}\{x\} \times D_{i}(x) .
$$

First note that $Z=\bigcap_{i=1}^{\infty} Z^{i}$. Also each nonempty section $\left(Z^{i}\right)_{x}$ of $Z^{i}$ is equal to $D_{i}(x) \in G_{r_{i}}$. Hence, by the induction hypothesis, $Z^{i} \in(V G)_{r_{i}}$, for each $i$, and therefore

$$
Z=\bigcap_{i=1}^{\infty} Z^{i} \in(V G)_{\alpha},
$$

by the definition of the family $(V G)_{\alpha}$.

Case 3. $\alpha$ is odd and greater than 1.

For each $x \in$ domain $Z$, let $\left\{D_{i}(x)\right\}_{i=1}^{\infty}$ be a sequence of members of $G_{\alpha-1}$ for which $Z_{x}=\bigcup_{i=1}^{\infty} D_{\imath}(x)$, and let $Z^{i}=\bigcup_{x \in \text { domain }(Z)}\{x\} \times D_{\imath}(x)$, for each $i$.

Again it follows that $Z^{i} \in G_{\alpha-1}$, for each $i$, and

$$
Z=\bigcup_{i=1}^{\infty} Z^{i} \in(V G)_{\alpha}
$$

COROLlary 4. If $Z \cong X \times X$ is the graph of a function then $Z \in \mathscr{R} \subseteq \mathscr{\mathscr { B }}(\mathscr{R})$.

Proof. Let $G$ be a countable basis for the real topology and note that, for each $x \in X, Z_{x}$ is a singleton and hence $Z_{x} \in G_{2}$. Thus by Theorem $3, Z \in(V G)_{2} \subseteq \mathscr{R} 2 \subseteq \mathscr{B}(\mathscr{R})$. Also see [7].

THEOREM 5. Let $X$ be the real numbers and let $G$ be a countable base for the usual topology on $X$. The following three statements are equivalent:

(1) $\mathrm{CH}$ holds

(2) if $Z \subseteq X \times X$, then $Z=A \cap B$, where $A \in(V G)_{2}$ and $B \in(H G)_{2}$ and

(3) if $E \leqq X \times X$, then $E=C \cup D$, where $C \in \mathscr{P}(V G)$ and $D \in$ $\mathscr{B}(H G)$. 
Proof. First, assume $\mathrm{CH}$ and suppose $Z \cong X \times X$. As is well known [7], the complement of $Z$ is the union of two sets $H$ and $K$ such that each vertical section of $H$ is countable and each horizontal section of $K$ is countable.

Let $A$ be the complement of $H$ and let $B$ be the complement of $K$. Then each vertical section of $A$ is a $G_{2}$ set and by Theorem 3, $A \in(V G)_{2}$. Similarly, $B \in(H G)_{2}$. Of course, $Z=A \cap B$.

Since $A \in(V G)_{2} \leqq \mathscr{R}_{2}$ and $B \in(H G)_{2} \subseteq \mathscr{R}_{2}$ and $\mathscr{R}_{2}$ is closed under finite intersections, $Z \in \mathscr{R}_{2}$. Thus, if $\mathrm{CH}$ holds, then the order of $\mathscr{R}$ is $\leqq 2$. Since the graph of the identity function, $f(x)=x$, is not in $\mathscr{R}$, it follows that the order of $\mathscr{R}$ is 2 .

Now, suppose statement 2 holds and $E \subseteq X \times X$. Then, the complement of $E$ can be expressed as the intersection of sets $A$ and $B$ with $A \in(V G)_{2}$ and $B \in(H G)_{2}$. It follows that $A^{\prime} \in(V G)_{3} \leqq \mathscr{P}(V G)$ and $B^{\prime} \in(H G)_{3} \subseteq \mathscr{B}(H G)$. Thus, $E$ is the union of two sets $C$ and $D$, where $C \in \mathscr{B}(V G)$ and $D \in \mathscr{B}(H G)$.

Finally, assume statement 3 holds. Let $T$ be a totally imperfect subset of $X$ of cardinality $c$. The existence of such a set can be proven without assuming $\mathrm{CH}[3, \mathrm{p}$. 514]. Let $E=T \times T$ and let $E=C \cup D$, with $C \in \mathscr{B}(V G)$ and $D \in \mathscr{B}(H G)$. Then each vertical section of $C$ is a subset of $T$ which is a Borel set. Since an uncountable Borel set contains a perfect set and $T$ contains no perfect set, we have that each vertical section of $C$ is countable. Similarly, each horizontal section of $D$ is countable. But, as is well known [10] this implies $\mathrm{CH}$.

This completes the proof of Theorem 5 .

The following two lemmas are well known.

LEMMA 6. If $F$ is a family of sets, $\alpha$ is a countable ordinal, and $A \in F_{\alpha}$, then there is a countable subfamily $J$ of $F$ for which $A \in J_{\alpha}$.

LeMma 7. If $F$ is a family of sets, $\mathscr{C}(F) \subseteq F$, and $A \in \mathscr{B}(F)$ then there is a countable subfamily $J$ of $F$ and a countable ordinal $\alpha$ for which $A \in J_{\alpha}$.

THEOREM 8. (a) The following two statements are equivalent:

(i) For each subset $Z$ of $X \times X$ there is a countable ordinal $\alpha$ such that $Z \in \mathscr{R}_{\alpha}$.

(ii) If $H$ is a family of subsets of $X$ and Card $H=\operatorname{Card} X$, then there is a countable family $G$ of subsets of $X$ and a countable ordinal $\alpha$ for which $H \subseteq G_{\alpha}$.

(b) If $\alpha$ is a countable ordinal, the following two statements are equivalent:

(i) Each subset of $X \times X$ is a member of $\mathscr{R}_{\alpha}$. 
(ii) If $H$ is a family of subsets of $X$ and Card $H=$ Card $X$ then there is a countable family $G$ of subsets of $X$ for which $H \subseteq G_{\alpha}$.

Proof. The proof of part (b) is similar to that of part (a) which is given below.

First suppose (i) holds, and suppose that $H$ satisfies the hypotheses of (ii). Define the subset $Z \cong X \times X$ by letting each member of $H$ be a vertical section of $Z$. More precisely, let $f$ be a 1-1 function from $X$ to $H$ and let

$$
Z=\bigcup_{x \in X}\{x\} \times f(x) .
$$

By (i) there is a countable ordinal $\alpha$ such that $Z \in \mathscr{R}_{\alpha}$ and hence by Lemma 6 , there is a countable subfamily $J$ of $\mathscr{R}$ for which $Z \in J_{\alpha}$. Let

$$
G=\{B: A \times B \in J\},
$$

note that $Z \in(V G)_{\alpha}$ and use Theorem 3 to conclude that $H \subseteq G_{\alpha}$.

Now suppose (ii) holds, and that $Z \subseteq X \times X$. Let $H$ be the family of vertical sections of $Z$, and use (ii) to secure a countable family $G$ and a countable ordinal $\alpha$ for which $H \subseteq G_{\alpha}$. Thus $Z_{x} \in G_{\alpha}$ for each $x \in$ domain $Z$ and by Theorem 3

$$
Z \in(V G)_{\alpha} \subseteq \mathscr{R}_{\alpha} .
$$

THEOREM 9. The following four statements are equivalent:

(i) Each subset of $X \times X$ is a member of $\mathscr{B}(\mathscr{R})$.

(ii) If $H$ is a family of subsets of $X$ and Card $H=$ Card $X$ then there is a countable family $G$ and a countable ordinal $\alpha$ for which $H \cong G_{\alpha}$.

(iii) There is a countable ordinal $\alpha$ such that, for each family $H$ of subsets of $X$ with Card $H=\operatorname{Card} X$, there is a countable family $G$ for which $H \subseteq G_{\alpha}$.

(iv) There is a countable ordinal $\alpha \geqq 2$ such that each subset of $X \times X$ is a member of $\mathscr{R}_{\alpha}$.

Proof. Statements (i) and (ii) are equivalent by Lemma 7 and Theorem 8a. Clearly (iii) implies (ii) and (iv) implies (i). Also by Theorem $8 \mathrm{~b}$ it follows that (iii) implies (iv). $\alpha$ cannot be equal to 1 in (iv) because by (i) the identity function $f(x)=x$ is not in $\mathscr{R}_{1}$.

We complete the proof by showing that (ii) implies (iii). Since this result is immediate if $X$ is countable we will assume that Card $X \geqq \omega_{1}$.

Suppose that (ii) holds and that (iii) does not. Then for each $\alpha<\omega_{1}$, there is a family $H(\alpha)$ of subsets of $X$ for which Card $H(\alpha)=$ 
Card $X$ and

for each countable $G, H(\alpha) \nsubseteq G_{\alpha}$.

Let $H^{\prime}=\bigcup_{\alpha<\omega_{1}} H(\alpha)$. Thus Card $H^{\prime}=$ Card $X$ and hence by (ii) there is a countable family $G^{\prime}$ and a countable ordinal $\alpha^{\prime}$ for which $H^{\prime} \subseteq G_{\alpha^{\prime}}^{\prime}$. But then $H\left(\alpha^{\prime}\right) \subseteq H^{\prime} \subseteq G_{\alpha^{\prime}}^{\prime}$ in contradiction of (1).

Therefore (ii) implies (iii).

In part (ii) above the family $G$ can be chosen so that $G_{\omega_{1}}$ is closed to complementation (i.e., is a $\sigma$-algebra).

In view of condition (ii) of Theorem 9 , it is interesting to note that R. Mansfield has shown that if $G$ is a countable family of Lebesgue measurable sets, then $B(G)$ does not contain all analytic sets [5].

As was mentioned in the introduction it would be interesting to know whether the formula " $H \subseteq G_{\alpha}$ " in Theorem 9 could be replaced by $H \subseteq \mathscr{B}(G)$. We do not know the answer to this question.

THeOREm 10. If $\mathrm{CH}$ holds, Card $X=c, H$ is a family of subsets of $X$, and Card $H=c$, then there is a countable family $G$ for which $H \subseteq G_{2}$.

Proof. By Theorem 5 each subset $Z$ of $X \times X$ is a member of $\mathscr{R}_{2}$. The desired conclusion now follows from Theorem $8 \mathrm{~b}$.

4. Generating Borel sets. Let $R$ be the set of reals, and let $H$ be the family of all Borel subsets of $R$. This family has cardinality c. Suppose $G$ is a countable family of subsets of $R$ such that $H \cong G_{\omega_{1}}$ and $G_{\omega_{1}}$ is closed to complementation. The next two theorems show that, even if the family $G$ generates all the Borel sets at an early stage, the order of $G$ is $\omega_{1}$. This is a generalization of the well known result $[4,9]$ that if $G$ is a countable basis for the real topology then $G$ has order $\omega_{1}$. Our proof which is a usual "diagonal" type argument, parallels somewhat Lebesgue's proof of that result [3, p. 368].

Let $G=\left\{V_{1}, V_{2}, V_{3}, \cdots\right\}$, let $N$ be the set of irrational numbers between 0 and 1 and let $K$ be the family $\left\{\theta_{1}, \theta_{2}, \theta_{3}, \cdots\right\}$ of all intersections of the members of $G$ with $N$,

$$
\theta_{i}=V_{i} \cap N \text {. }
$$

It will be shown that the order of $K$ is $\omega_{1}$. It then follows that the order of $G$ is $\omega_{1}$.

For each $z \in N$, let $\left(z_{1}, z_{2}, z_{3}, \cdots\right)$ be the sequence of integers appearing in the continued fraction expansion of $z$. This defines a 
reversible transformation from $N$ onto the set of all sequences of positive integers. Let

$$
\begin{aligned}
z^{1} & =\left(z_{1}, z_{3}, z_{5}, \cdots\right) \quad \text { (odd indices) } \\
z^{2} & =\left(z_{2}, z_{6}, z_{10}, \cdots\right) \\
z^{3} & =\left(z_{4}, z_{12}, z_{20}, \cdots\right) \\
\vdots & \\
z^{n} & =\left(z_{2^{n-1}}, z_{3 \cdot 2^{n-1}}, z_{5 \cdot 2^{n-1}}, \cdots\right)
\end{aligned}
$$

This defines a homeomorphism between $N$ and $N^{\boldsymbol{\aleph}_{0}}$ (see Kuratowski [3], p. 369). Also note that if $f$ is a continuous function from $N$ into $N$, then the functions $f_{n}$ from $N$ into the space of positive integers are continuous, where

$$
f(z)=\left(f_{1}(z), f_{2}(z), f_{3}(z), \cdots\right), \quad \text { or } \quad\left(f_{n}(z)=f(z)_{n}\right) .
$$

Recall that $K=\left\{\theta_{1}, \theta_{2}, \theta_{3}, \cdots\right\}$. The family $K_{\alpha}$ which appears in Theorem 11 is defined in $\S 2$.

THEOREM 11. For each countable ordinal $\alpha, \alpha>0$, there is a function $U_{\alpha}$ from $N$ onto $K_{\alpha}$ such that if $f$ is a continuous function from $N$ into $N$, then the set

$$
A_{f}=\left\{z: z \in U_{\alpha}(f(z))\right\}
$$

is in $\mathscr{B}(K)$.

Proof. Let $U_{1}(z)=\bigcup_{n=1}^{\infty} \theta_{z_{n}}$, for each $z \in N$. Clearly $U_{1}$ maps $N$ onto $K_{1}$.

Let $f$ be a continuous function from $N$ onto $N$. We have

$$
\begin{aligned}
A_{f} & =\left\{z: z \in U_{1}(f(z))\right\} \\
& =\left\{z: z \in \bigcup_{n=1}^{\infty} \theta_{f_{n}(z)}\right\} \\
& =\bigcup_{n=1}^{\infty}\left\{z: z \in \theta_{f_{n}(z)}\right\} .
\end{aligned}
$$

For each $n$,

$$
\left\{z: z \in \theta_{f_{n}(z)}\right\}=\bigcup_{i=1}^{\infty}\left\{J_{n_{i}} \cap \theta_{i}\right\}
$$

where $J_{n_{i}}=\left\{z: f_{n}(z)=i\right\}$. Since each $f_{n}$ is continuous it follows that each $J_{n_{i}}$ is open and therefore the set $A_{f}$ belongs to $G_{\omega_{1}}$.

Suppose $1<\alpha<\omega_{1}$ and suppose that the function $U_{\gamma}$ has been defined for each ordinal $\gamma$ with $1 \leqq \gamma<\alpha$. (Induction hypothesis.)

If $\alpha$ is odd, let 


$$
U_{\alpha}(z)=\bigcup_{n=1}^{\infty} U_{c-1}\left(z^{n}\right), \quad \text { for } \quad z \in N
$$

Clearly $U_{\alpha}$ maps $N$ onto $K_{\alpha}$.

If $\alpha$ is even, let $\left\{\gamma_{n}\right\}_{n=1}^{\infty}$ be a sequence of odd ordinals less than $\alpha$ such that each odd ordinal less than $\alpha$ appears infinitely often in $\left\{\gamma_{i}\right\}_{n=1}^{\infty}$ and let

$$
U_{\alpha}(z)=\bigcap_{n=1}^{\infty} U_{r_{n}}\left(z^{n}\right), \text { for } \quad z \in N
$$

If $A \in K_{\alpha}$ ( $\alpha$ is still even), then

$$
A=\bigcap_{n=1}^{\infty} D_{n}
$$

where $D_{n} \in K_{\gamma_{n}}$, for each $n$. For each $n$, let $y_{n}$ be a point of $N$ such that

$$
D_{n}=U_{r_{n}}\left(y_{n}\right) \text {. }
$$

And let $z$ be the point mapped by the transformation described by (*) to the point $\left(y_{1}, y_{2}, y_{3}, \cdots\right)$ of $N^{*_{0}}$. Thus

$$
U_{\alpha}(z)=A
$$

and $U_{\alpha}$ maps $N$ onto $K_{\alpha}$.

This completes the definition of the functions $U_{\alpha}$. Now let $f$ be a continuous function from $N$ into $N$. It will be shown that if $\alpha$ is even the set

$$
A_{f}=\left\{z: z \in U_{\alpha}(f(z))\right\}
$$

is in $G_{\omega_{1}}$. The argument for the case $\alpha$ is odd is similar.

We have

$$
\begin{aligned}
A_{f} & =\left\{z: z \in \bigcap_{n=1}^{\infty} U_{r_{n}}\left((f(z))^{n}\right)\right\} \\
& =\bigcap_{n=1}^{\infty}\left\{z: z \in U_{r_{n}}\left((f(z))^{n}\right)\right\} .
\end{aligned}
$$

But, for each $n$, the function $z \rightarrow(f(z))^{n}$, being the composition of two continuous functions, is a continuous function from $N$ to $N$.

Thus by the induction hypothesis, the sets $\left\{z: z \in U_{r_{n}}\left((f(z))^{n}\right)\right\}$ are in the family $G_{\omega_{1}}$. Therefore $A_{f} \in G_{\omega_{1}}$.

THEOREM 12. If $G$ is a countable family of subsets of real numbers with $\mathscr{C}(G) \subseteq G$, and each Borel set is a member of $\mathscr{B}(G)$ then $G$ has order $\omega_{1}$. 
Proof. Let $\alpha$ be any countable ordinal, and let

$$
I_{\alpha}=\left\{z: z \notin U_{\alpha}(z)\right\}
$$

Suppose $I_{\alpha} \in K_{\alpha}$, and let $U_{\alpha}(z)=I_{\alpha}$. If $z \in I_{\alpha}$ then $z \in U_{\alpha}(z)$. But this contradicts the definition of $I_{\alpha}$. If $z \notin I_{\alpha}$, then $z \in U_{\alpha}(z)=I_{\alpha}, z \in I_{\alpha}$. This contradiction shows that $I_{\alpha} \notin K_{\alpha}$.

Since $\mathscr{B}(G)=G_{\omega_{1}}$ (because $\left.\mathscr{C}(G) \subseteq G\right)$, and $I_{\alpha}^{\prime}=\left\{z: z \in U_{\alpha}(z)\right\} \in G_{\omega_{1}}$ by Theorem 11, it follows that $I_{\alpha} \in G_{\omega_{1}}-G_{\alpha}$. Thus $G_{\alpha} \neq G_{\omega_{1}}$, and hence $G$ has order $\omega_{1}[3$, p. 371].

\section{REFERENCES}

1. J. A. Johnson, Amer. Math. Monthly, 79 (1972), 307.

2. Kenneth Kunen, Inaccessibility Properties of Cardinals, Ph. D. Thesis, Department of Mathematics, Stanford University, August, 1968.

3. C. Kuratowski, Topology I, Academic Press, New York, 1966.

4. H. Lebesgue, Sur les fonctions representable analytiquement, J. de Math., (6) 1 (1905), 139-216.

5. D. A. Martin and R. M. Solovay, Internal Cohen extensions, Annals of Math. Logic, 2 (1970), 143-148.

6. B. V. Rao, On discrete Borel spaces and projective sets, Bull. Amer. Math. Soc., 75 (1969), 614.

7. 197.

8. W. Sierpinski, Sur l'existence de diverses classes d'ensembles, Fund. Math., 14 (1929), $82-91$.

9. —_, Hypothèse du Continu, Warsaw, 1934, 9.

Received November 14, 1972 and in revised form July 12, 1973. This work was partially supported by NSF Grant GJ-32269.

UNIVERSITY OF WISCONSIN

UNIVERSITY OF TEXAS

AND

UNIVERSITY OF FLORIDA 


\section{PACIFIC JOURNAL OF MATHEMATICS}

\section{EDITORS}

RICHARD ARENS (Managing Editor)

University of California

Los Angeles, California 90024

R. A. BeAumont

University of Washington

Seattle, Washington 98105
J. DugundJI*

Department of Mathematics

University of Southern California

Los Angeles, California 90007

D. Gilbarg and J. Milgram

Stanford University

Stanford, California 94305

\section{ASSOCIATE EDITORS}

E. F. BECKENBACH

B. H. NEUMANN

F. WOLF

K. YoSHIDA

\section{SUPPORTING INSTITUTIONS}

UNIVERSITY OF BRITISH COLUMBIA
CALIFORNIA INSTITUTE OF TECHNOLOGY
UNIVERSITY OF CALIFORNIA
MONTANA STATE UNIVERSITY
UNIVERSITY OF NEVADA
NEW MEXICO STATE UNIVERSITY
OREGON STATE UNIVERSITY
UNIVERSITY OF OREGON
OSAKA UNIVERSITY

UNIVERSITY OF BRITISH COLUMBIA CALIFORNIA INSTITUTE OF TECHNOLOGY UNIVERSITY OF CALIFORNIA MONTANA STATE UNIVERSITY NEW MEXICO STATE UNIVERSITY UNIVERSITY OF OREGON OSAKA UNIVERSITY
UNIVERSITY OF SOUTHERN CALIFORNIA STANFORD UNIVERSITY UNIVERSITY OF TOKYO UNIVERSITY OF UTAH WASHINGTON STATE UNIVERSITY UNIVERSITY OF WASHINGTON AMERICAN MATHEMATICAL SOCIETY NAVAL WEAPONS CENTER

* C. R. DePrima California Institute of Technology, Pasadena, CA 91109, will replace J. Dugundji until August 1974. 


\section{Pacific Journal of Mathematics}

\section{Vol. 51, No. $1 \quad$ November, 1974}

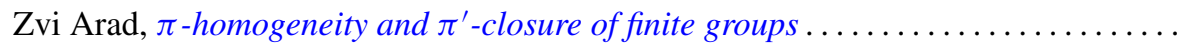

Ivan Baggs, A connected Hausdorff space which is not contained in a maximal

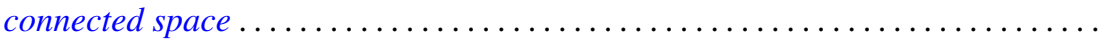

Eric Bedford, The Dirichlet problem for some overdetermined systems on the unit ball in $C^{n}$

R. H. Bing, Woodrow Wilson Bledsoe and R. Daniel Mauldin, Sets generated by

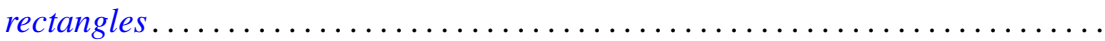

Carlo Cecchini and Alessandro Figà-Talamanca, Projections of uniqueness for

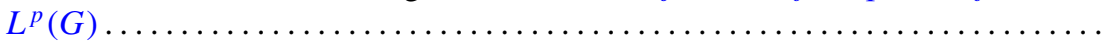

Gokulananda Das and Ram N. Mohapatra, The non absolute Nörlund summability of Fourier series .

Frank Rimi DeMeyer, On separable polynomials over a commutative ring ........ Richard Detmer, Sets which are tame in arcs in $E^{3} \ldots \ldots \ldots \ldots \ldots \ldots \ldots \ldots$

William Erb Dietrich, Ideals in convolution algebras on Abelian groups ..........

Bryce L. Elkins, A Galois theory for linear topological rings .................

William Alan Feldman, A characterization of the topology of compact convergence on $C(X)$.

Hillel Halkin Gershenson, A problem in compact Lie groups and framed cobordism

Samuel R. Gordon, Associators in simple algebras.

Marvin J. Greenberg, Strictly local solutions of Diophantine equations

Jon Craig Helton, Product integrals and inverses in normed rings . . . . . . . . . . . .

Domingo Antonio Herrero, Inner functions under uniform topology . . .

Jerry Alan Johnson, Lipschitz spaces .

Marvin Stanford Keener, Oscillatory solutions and multi-point boundary value

functions for certain nth-order linear ordinary differential equations.

John Cronan Kieffer, A simple proof of the Moy-Perez generalization of the

Shannon-McMillan theorem .......................

Joong Ho Kim, Power invariant rings

Gangaram S. Ladde and V. Lakshmikantham, On flow-invariant sets .

Roger T. Lewis, Oscillation and nonoscillation criteria for some self-adjoint even

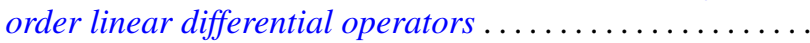

Jürg Thomas Marti, On the existence of support points of solid convex sets ..

John Rowlay Martin, Determining knot types from diagrams of knots . .

James Jerome Metzger, Local ideals in a topological algebra of entire functions

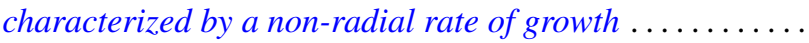

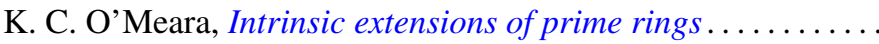

Stanley Poreda, A note on the continuity of best polynomial approximations ..

Robert John Sacker, Asymptotic approach to periodic orbits and local prolongations

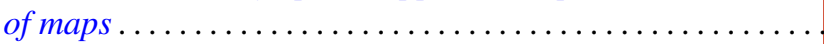

Eric Peter Smith, The Garabedian function of an arbitrary compact set . .

Arne Stray, Pointwise bounded approximation by functions satisfying a side condition

John St. Clair Werth, Jr., Maximal pure subgroups of torsion complete abelian

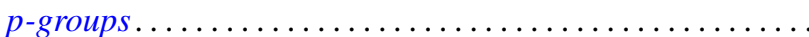

